
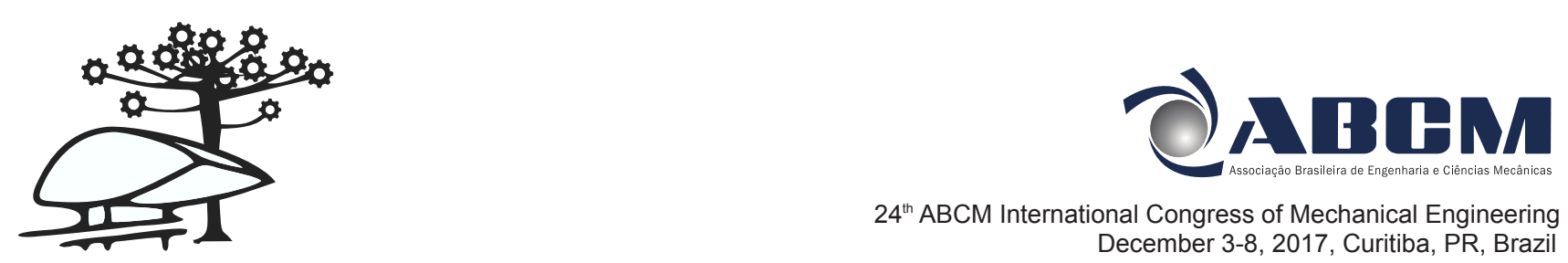

$24^{\text {th }} \mathrm{ABCM}$ International Congress of Mechanical Engineering

December 3-8, 2017, Curitiba, PR, Brazil

24th COBEM - 2017

\title{
COBEM-2017-1369 \\ A STUDY OF THE ERRORS IN SWING-BY DESIGN BY THE "PATCHED-CONICS" APPROACH APPLIED TO THE GALILEAN MOONS
}

\section{Rodolfo Batista Negri}

Instituto Nacional de Pesquisas Espaciais

rodolfo.negri@inpe.br

Antonio Fernando Bertachini de Almeida Prado

Instituto Nacional de Pesquisas Espaciais

antonio.prado@inpe.br

Abstract. The simplest method to design a swing-by is the well known "patched-conics" approach. However this approach assumes some simplifications as: i) there is no perturbation on the trajectory during the maneuver; ii) the maneuver is instantaneous. For most of the cases these assumptions are appropriate and bring only small errors. However, there are some cases where the errors are considerably large. Some situations where a better understanding of the associated errors are desirable. Therefore, this work intend to attend this demand by mapping the errors in $\Delta V s$ given by the "patchedconics" approach. In order to do so, the values obtained by a three dimensional "patched-conics" approach is compared with values obtained from a circular restricted three-body problem simulation, considered as the real ones, for the same initial conditions. In view of a relatively great number of missions in the next years aiming to explore the Galilean moons of Jupiter - e.g., Europa Clipper, Juice and so on - this work will focus its analysis on them.

Keywords: patched-conics, swing-by, gravity-assisted, restricted three body problem, Jupiter system

\section{INTRODUCTION}

The main aspects of a swing-by maneuver can be described using the "patched-conics" model, which splits the problem in a sequence of "two-body problems", as studied by Broucke (1988), for the planar motion, and Prado (2000), for the three dimensional maneuver. A first attempt to measure the differences between the two models, in terms of the variation of energy, is made by Prado (2007), which compared both approaches when applied to a planar maneuver. Negri et al. (2017) expanded this work to the three dimensional maneuver. There are also some studies of this problem related to astronomy, as the work of Greenberg et al. (1988). The goals are different, but the physical explanations are the same.

So, the present paper focus in appliying the work done by Negri et al. (2017) to the Galilean moons of Jupiter. A spacecraft is assumed to make a close approach with one of the moons, but its motion is not restricted to the orbital plane of the primaries. Hence, the location of the periapsis needs an extra angle to indicate the out-of-plane component of its position in space. The velocity at periapsis also needs a new variable, which can be an angle that defines its direction in space. So, the new number of parameters is increased to five, by adding those two new ones to the well known periapsis distance $r_{p}$, magnitude of the velocity at periapsis $v_{p}$ and angle of approach $\alpha$ (Broucke, 1988).

Therefore, the strategy used in the present paper is to define a swing-by trajectory in the three-dimensional space by specifying those five parameters. But as it is done by Negri et al. (2017) for some selected systems of the solar system, it is calculated the $\Delta V$ instead of energy, using the "patched-conics" approach and the "restricted three-body problem". It is assumed that the results coming from the restricted problem represent the "real values" and the results coming from the "patched-conics" are the "estimated values". So, by varying the five parameters, it is possible to measure the effects of each parameter in the errors of the "estimated maneuver". The goal is to give estimates of the errors for the Galilean moons of Jupiter, since they will be very important in the next decades of space exploration (Grasset et al., 2013; Phillips and Pappalardo, 2014; Bolton, 2010), so it is possible to know in advance if it is possible to use the "patched-conics" model, considering the system under study and the accuracy required by the maneuver. In order to do so, it is measured the errors as a function of all the parameters that define the maneuver. Minimum and maximum errors are shown as a function of each parameter and plotted.

\section{MATHEMATICAL MODELS AND METHODS}

In this section are presented the mathematical models and the methods used in this work. 


\subsection{Stating the problem}

The system is composed by three point masses, where two of them are called $M_{1}$ and $M_{2}$. The first body, $M_{1}$, has the largest mass, while $M_{2}$ is the second largest body in mass. Both are from now on called primaries, and each of them perform a circular orbit around their center of mass. The third body is the spacecraft, which has negligible mass if compared to the other two.

For a better understanding of the problem is convenient to define three reference frames. They are shown in Fig. 1 and called: synodic reference frame, or $x y z$; inertial reference frame, or $X Y Z$; and the pseudo-inertial reference frame, or $X^{\prime} Y^{\prime} Z^{\prime}$.

The synodic reference frame $x y z$, showed in Fig. 1(a), is the reference frame usually used in the circular restricted three-body problem. As the name points out, it is a rotating system, and has a constant angular speed represented by $n$. Its origin is in the center of mass of the system. Its $x$ axis matches exactly with the line connecting the primaries, while its $y$ axis lies on the orbital plane of the primaries, perpendicular to the $x$ axis. Lastly, the $z$ axis completes the right-handed coordinate system.

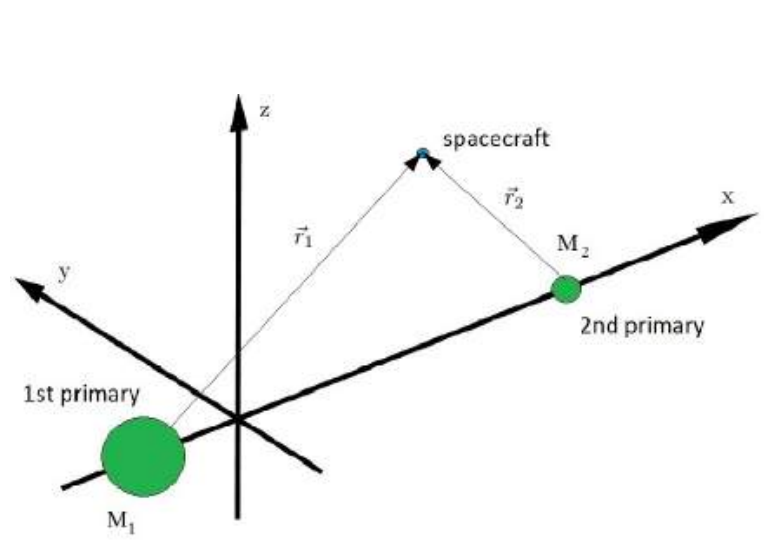

(a) Synodic reference system, $x y z$.

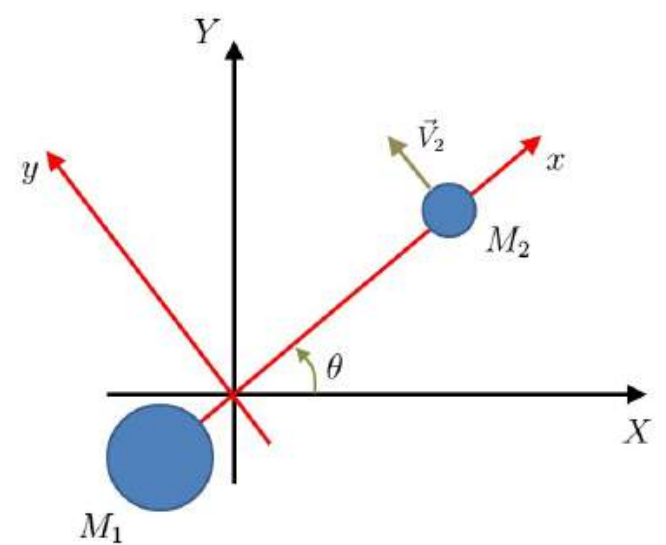

(b) Inertial reference system, $X Y Z$.

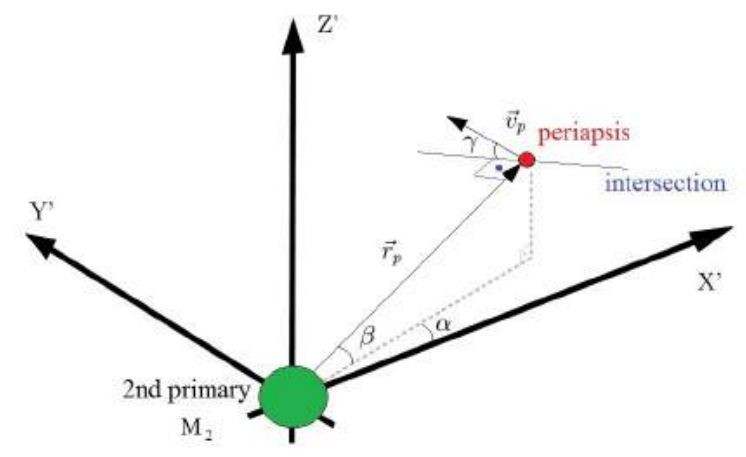

(c) Representation of the parameters that define the swing-by in the pseudo-inertial reference system $X^{\prime} Y^{\prime} Z^{\prime}$.

Figure 1. The used reference systems.

We can move now to the inertial coordinate system $X Y Z$, presented in Fig. 1(b). This is a fixed reference frame with origin in the center of mass of the system, it is defined in such a way that its axis are coincident with the synodic system axis at the initial time $(t=0)$.

The third reference system to be presented is the pseudo-inertial $X^{\prime} Y^{\prime} Z^{\prime}$, as shown by Fig. 1(c). Its axis points to the same direction of the $X Y Z$ system axis, however the system is displaced and moves coupled to $M_{2}$, that is why it is called pseudo-inertial. The swing-by is performed on the second primary $\left(M_{2}\right)$, thus the $X^{\prime} Y^{\prime} Z^{\prime}$ system is convenient to define the five parameters which univocally define the swing-by.

These parameters define the position and velocity of the spacecraft at the periapsis of the spacecraft approach with $M_{2}$, and they are also presented in Fig. 1(c), where: $r_{p}$ is the distance between the periapsis and the center of the second primary; $\alpha$ and $\beta$ are the angles defining the postion $\overrightarrow{r_{p}}$ in the three dimensional space; $v_{p}$ is the speed of the spacecraft at the periapsis with respect to $M_{2}$; and $\gamma$ is the angle between the velocity $\vec{v}_{p}$ and the intersection between a plane that contains the periapsis and is parallel to the $X^{\prime} Y^{\prime}$ plane and a plane perpendicular to $r_{p}$ that contains $v_{p}$. Therefore, the 
components of position and velocity in the $X^{\prime} Y^{\prime} Z^{\prime}$ reference system can be written as (Prado and de Felipe, 2007):

$$
\begin{aligned}
X^{\prime} & =r_{p} \cos \beta \cos \alpha \\
Y^{\prime} & =r_{p} \cos \beta \sin \alpha, \\
Z^{\prime} & =r_{p} \sin \beta \\
\dot{X}^{\prime} & =-v_{p} \sin \gamma \sin \beta \cos \alpha-v_{p} \cos \gamma \sin \alpha, \\
\dot{Y}^{\prime} & =-v_{p} \sin \gamma \sin \beta \sin \alpha+v_{p} \cos \gamma \cos \alpha, \\
\dot{Z}^{\prime} & =v_{p} \cos \beta \sin \gamma .
\end{aligned}
$$

\subsection{The restricted problem}

Firstly, it is necessary to write the Eq. 1 to Eq. 6 in the $x y z$ reference system, they are:

$$
\begin{aligned}
x & =1-\mu+r_{p} \cos \beta \cos \alpha, \\
y & =r_{p} \cos \beta \sin \alpha, \\
z & =r_{p} \sin \beta, \\
\dot{x} & =-v_{p} \sin \gamma \sin \beta \cos \alpha-v_{p} \cos \gamma \sin \alpha+r_{p} \cos \beta \sin \alpha, \\
\dot{y} & =-v_{p} \sin \gamma \sin \beta \sin \alpha+v_{p} \cos \gamma \cos \alpha-r_{p} \cos \beta \cos \alpha, \\
\dot{z} & =v_{p} \cos \beta \sin \gamma .
\end{aligned}
$$

Now is presented the numerical steps utilized to solve the circular restricted three-body equations of motion ${ }^{1}$. The equations of motion are (Szebehely, 1967):

$$
\begin{aligned}
\ddot{x}-2 \dot{y} & =x-(1-\mu) \frac{x+\mu}{r_{1}^{3}}-\mu \frac{x-1+\mu}{r_{2}^{3}}, \\
\ddot{y}+2 \dot{x} & =y-(1-\mu) \frac{y}{r_{1}^{3}}-\mu \frac{y}{r_{2}^{3}}, \\
\ddot{z} & =-(1-\mu) \frac{z}{r_{1}^{3}}-\mu \frac{z}{r_{2}^{3}} ;
\end{aligned}
$$

where $r_{1}$ and $r_{2}$ are, respectively, the distance between the spacecraft and the first and second primary, as shown in Fig. 1(a).

In order to find the variation of velocity given by the restricted problem $\left(\Delta V_{R P}\right)$, the following algorithm is used:

- By using Eq. 7 to Eq. 12, with initial values for $\alpha, \beta, r_{p}, \gamma$ and $v_{p}$, the initial conditions are calculated;

- The equations of motion are integrated forward in time until the spacecraft reaches a distance $r_{2}$ equal to the sphere of influence (SOI) of $M_{2}\left(r_{S O I}\right)$, which the radius is given by (Araujo et al., 2008):

$$
r_{S O I}=\left(\frac{\mu}{1-\mu}\right)^{2 / 5} \text {. }
$$

- Once the integration is performed, the speed after the maneuver $V_{R P}^{+}$is calculated by saving the velocity in the $x y z$ reference system, transforming it to the $X Y Z$ reference system (Murray and Dermott, 1999) and obtaining its modulus;

- The spacecraft is then positioned again at its initial conditions, and the equations of motion are integrated backward in time, until $r_{2}$ reaches the sphere of influence of $M_{2}$ one more time;

- The speed $V_{R P}^{-}$before the swing-by is then calculated;

- Finally, the $\Delta V$ for the restricted problem is calculated as:

$$
\Delta V_{R P}=V_{R P}^{+}-V_{R P}^{-} .
$$

\footnotetext{
${ }^{1}$ A forth-order Runge-Kutta method with local truncation error to fifth-order was used to assure the precision and to adjust the step size.
} 


\subsection{The patched-conics approach}

For the patched-conics approach, the pseudo-inertial $X^{\prime} Y^{\prime} Z^{\prime}$ reference system is more convenient to describe the trajectory of the spacecraft as it performs the maneuver. Here, this system is assumed to be inertial, which means that no $M_{2}$ movement is taken into account. As a direct consequence, it is supposed that the maneuver is taken instantaneously.

Another assumption of this approach is that the restricted three-body problem may be reduced to a sequence of three two-body problems (spacecraft- $M_{1}$, spacecraft- $M_{2}$ and spacecraft- $M_{1}$ once again) that are patched together at its extremities (Minovitch, 2010). This means that the perturbation effects of $M_{2}$ in the orbit of the spacecraft around $M_{1}$ and the perturbation effects of $M_{1}$ in the orbit of the spacecraft around $M_{2}$ are not considered in this model. Therefore, there are three conic orbits. The points where the orbits are patched are determined by the SOI (Araujo et al., 2008) of $M_{2}$, which is given by Eq. 16.

By using this approach, Prado (2000) shows that the velocity after the maneuver $\left(\vec{V}_{i}\right)$ and before it $\left(\vec{V}_{o}\right)$, with respect to the $X Y Z$ reference frame, are calculated as:

$$
\begin{gathered}
\vec{V}_{i}=v_{\infty} \sin \delta\left[\begin{array}{c}
\cos \beta \cos \alpha \\
\cos \beta \sin \alpha \\
\sin \beta
\end{array}\right]+v_{\infty} \cos \delta\left[\begin{array}{c}
-\sin \gamma \sin \beta \cos \alpha-\cos \gamma \sin \alpha \\
-\sin \gamma \sin \beta \sin \alpha+\cos \gamma \cos \alpha \\
\cos \beta \sin \gamma
\end{array}\right]+\left[\begin{array}{c}
0 \\
V_{2} \\
0
\end{array}\right], \\
\vec{V}_{o}=-v_{\infty} \sin \delta\left[\begin{array}{c}
\cos \beta \cos \alpha \\
\cos \beta \sin \alpha \\
\sin \beta
\end{array}\right]+v_{\infty} \cos \delta\left[\begin{array}{c}
-\sin \gamma \sin \beta \cos \alpha-\cos \gamma \sin \alpha \\
-\sin \gamma \sin \beta \sin \alpha+\cos \gamma \cos \alpha \\
\cos \beta \sin \gamma
\end{array}\right]+\left[\begin{array}{c}
0 \\
V_{2} \\
0
\end{array}\right] .
\end{gathered}
$$

With some algebra is possible to show that the modulus of the vectors $\vec{V}_{i}$ and $\vec{V}_{o}$ are:

$$
\begin{aligned}
& V_{i}=\left[v_{\infty}^{2}+V_{2}^{2}+2 v_{\infty} V_{2}(\cos \alpha \cos \delta \cos \gamma+\cos \beta \sin \alpha \sin \delta-\cos \delta \sin \alpha \sin \beta \sin \gamma)\right]^{1 / 2} \\
& V_{o}=\left[v_{\infty}^{2}+V_{2}^{2}+2 v_{\infty} V_{2}(\cos \alpha \cos \delta \cos \gamma-\cos \beta \sin \alpha \sin \delta-\cos \delta \sin \alpha \sin \beta \sin \gamma)\right]^{1 / 2}
\end{aligned}
$$

where $V_{2}$ is the speed of $M_{2}$; the angles $\beta$ and $\alpha$ are in accordance with Fig. 1(c); $\delta$ is half of the turn angle of the trajectory of the spacecraft due to the passage by $M_{2}$, which can be expressed using two-body dynamics as (Broucke, 1988):

$$
\sin \delta=\frac{1}{1+r_{p} v_{\infty}^{2} / \mu},
$$

where $v_{\infty}$ is the speed of the spacecraft at the infinity with respect to $M_{2}$ (i.e., the $X^{\prime} Y^{\prime} Z^{\prime}$ reference system). From the two-body mechanics, $v_{\infty}$ is given by:

$$
v_{\infty}=\left(v_{p}^{2}-\frac{2 \mu}{r_{p}}\right)^{\frac{1}{2}} .
$$

Now, by using Eq. 20 and Eq. 21, it is possible to calculate the speed variation obtained by the swing-by, for the patched-conics approach, as:

$$
\Delta V_{P C}=V_{o}-V_{i}
$$

\section{RESULTS}

As made by Negri et al. (2017) for some systems of the solar system, this analysis is made using $\Delta V \mathrm{~s}$, in $\mathrm{km} / \mathrm{s}$, measured in the inertial reference frame $X Y Z$.

The error in $\Delta V$ is defined as:

$$
\Delta V_{\text {error }}=\Delta V_{R P}-\Delta V_{P C}
$$

From Fig. 2 to Fig. 8 is shown the maximum and minimum $\Delta V$ s applied to the Galilean moons to the whole set of parameters. They are standardized as follows: the (a) subfigures represent $\Delta V_{R P}$, the (b) represent $\Delta V_{P C}$ and the (c) represent $\Delta V_{\text {error }}$.

Starting by the analysis of the $\Delta V \mathrm{~s}$ under the influence of $r_{p}$, as shown in Fig. 2. As observed by Negri et al. (2017) larger $\Delta V$ s, Fig. 2(a) and Fig. 2(b), occurs for low $r_{p}$. The errors follow this same behaviour, as shown in Fig. 2(c).

Now, studying $v_{p}$ through $N$, as shown in Fig. 3. Analysing the $\Delta V$ s obtained by the restricted problem and by the patched-conics approach, Fig. 3(a) and Fig. 3(b), is noticeable a peak region for $\Delta V$. For instance, in the Fig. 3(b), this 
peak occurs between $N=1.2$ and $N=1.25$. This behaviour was expected, because Eq. 23, Eq. 20 and Eq. 21 show that the Eq. 24 has quadratic terms of $v_{p}$, which makes the relation between $\Delta V$ and $v_{p}$ a little more complex. The errors, Fig. 3(c), follows the same behaviour noted by Negri et al. (2017).

Moving now to the $\gamma$ angle, Fig. 4, and starting by the patched-conics approach, Fig. 4(b) shows that the maximum and minimum $\Delta V_{P C} \mathrm{~s}$ are constant, which might bring to the conclusion that $\Delta V_{P C}$ does not depend on $\gamma$. This was not expected, since Eq. 20 and Eq. 21 show the opposite, a clear dependence on $\gamma$. However, because our analysis is based on maximums and minimums, this figure might be misleading. The Fig. 4(d) disprove this conclusion by fixing all the parameters and varying only $\alpha$ and $\gamma$ applied to Ganymede-Jupiter and showing that $\Delta V_{P C}$ is not constant with the variation of the angle $\gamma$. The constant appearance in Fig. 4(b) is explained by the superposition of various different curves, for different sets of parameters, with equal peaks for different $\gamma$.

Figure 3(a) shows that the restricted problem does not follow the same behaviour. There is a clear advantage by using $\gamma= \pm 180^{\circ}$. This is a consequence that, differently of what occurs with the patched-conics approach, which assumes that the $X^{\prime} Y^{\prime} Z^{\prime}$ reference system is inertial, the restricted problem does not follows the same mistake. While the spacecraft performs its swing-by, $M_{2}$ keeps in its orbit around the center of mass of the system, making the velocity vector of $M_{2}$, $\vec{V}_{2}$, to be different at the inbound or outbound of the SOI. Figure 5 exemplifies it.

When the $\gamma$ angle assumes the value of $\pm 180^{\circ}$, it always acts in favour of increasing the effects of the maneuver over $\Delta V$, whether in the gain (when $180^{\circ}<\alpha<360^{\circ}$ ) or the loss (when $0^{\circ}<\alpha<180^{\circ}$ ) of energy. But when $\gamma$ assumes the value of $0^{\circ}$, this does not happen.

Figure 6 shows this behaviour. It represents a generic $M_{2}$ swing-by for the gain of energy as seem from the pseudoinertial reference frame $X^{\prime} Y^{\prime} Z^{\prime}$. But now, differently of what occurs in the patched-conics approach, this reference system is known as non-inertial and this is taken into account by changing $\vec{V}_{2}$ direction. One should notice that $\vec{v}_{\infty}$ acts in favour of $\vec{V}_{2}$ when $\gamma=180^{\circ}$, and the opposite when $\gamma=0^{\circ}$. The same effect is expected in the region of loss of energy, and now $\gamma= \pm 180^{\circ}$ should make $\vec{v}_{\infty}$ acts against $\vec{V}_{2}$, increasing the loss of speed.

Lastly, still in $\gamma$, Fig. 4(c) is coherent with Fig. 4(a) and Fig. 4(b).

The $\alpha$ angle, Fig. 7, has the same behaviour noticed by Negri et al. (2017). Figure 7(a) and Fig. 7(b) show that the maximum and minimum values occur at the expected $\alpha$ s. The maximum in $\alpha=270^{\circ}$ and minimum in $\alpha=180^{\circ}$. The errors follows this line as shown in Fig. 7(c).

The last parameter, $\beta$, is presented in Fig. 8. As expected, the Fig. 8(a) and Fig. 8(b) show larger $\Delta V \mathrm{~s}$ for $\beta=0^{\circ}$. The errors, Fig. 8(c), follows this behaviour as already noted by Negri et al. (2017).

Lastly, it must be highlighted the magnitude of the $\Delta V_{\text {error }}$ found and confirmed by all Figs. 2(c) to 8(c). They show errors of about $0.9 \mathrm{~km} / \mathrm{s}$ for the Io-Jupiter system, this is around $50 \%$ of the maximum value estimated by the patchedconics approach. For the Ganymede-Jupiter and Europa-Jupiter cases the error is approximately $0.6 \mathrm{~km} / \mathrm{s}$, reaching $40 \%$ of the estimated by the patched-conics approach for Ganymede and 60\% for Europa. Moreover, for Callisto, the maximum error is a bit greater than $0.4 \mathrm{~km} / \mathrm{s}$, which corresponds to around $40 \%$ of the estimated by the patched-conics. 


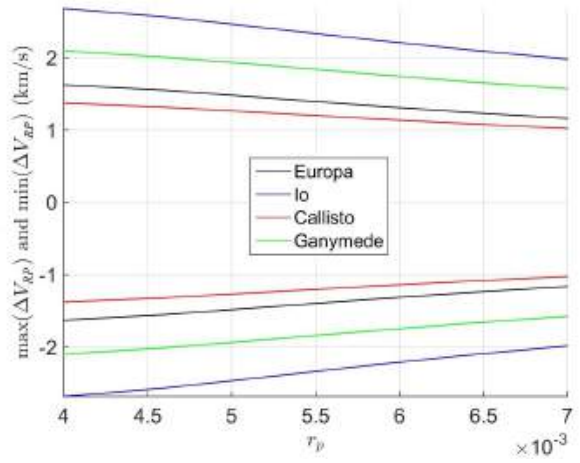

(a) $\Delta V_{R P}$

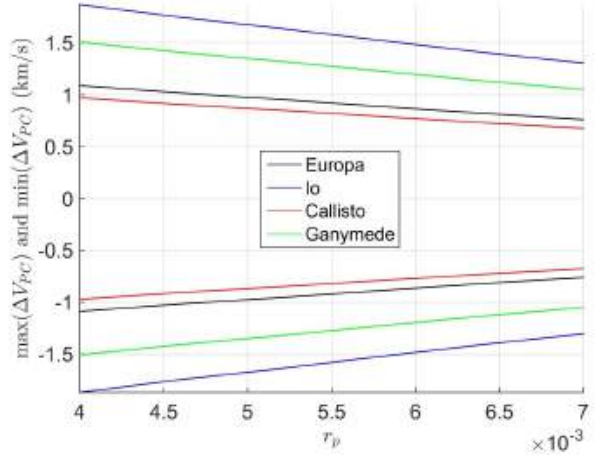

(b) $\Delta V_{P C}$

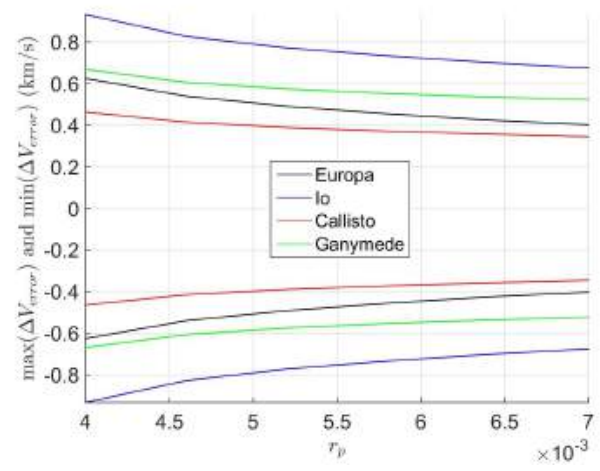

(c) $\Delta V_{\text {error }}$

Figure 2. Maximum and minimum $\Delta V \mathrm{~s}$ as function of $r_{p}$ applied to the Galilean moons.

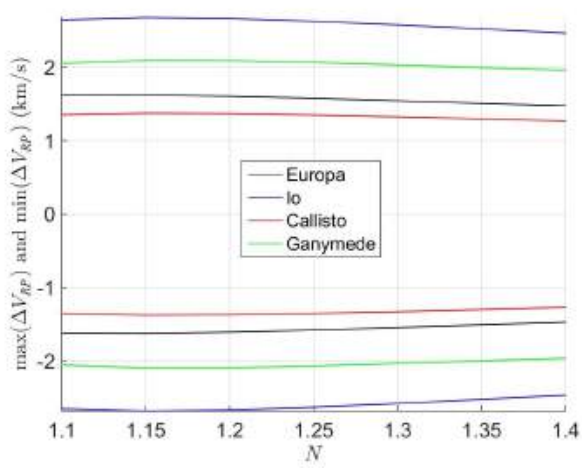

(a) $\Delta V_{R P}$

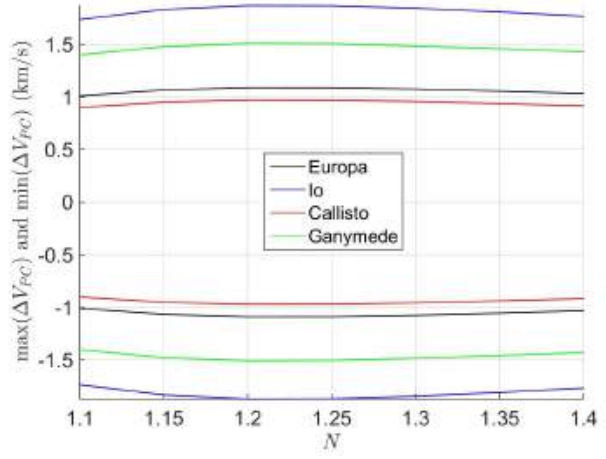

(b) $\triangle V_{P C}$

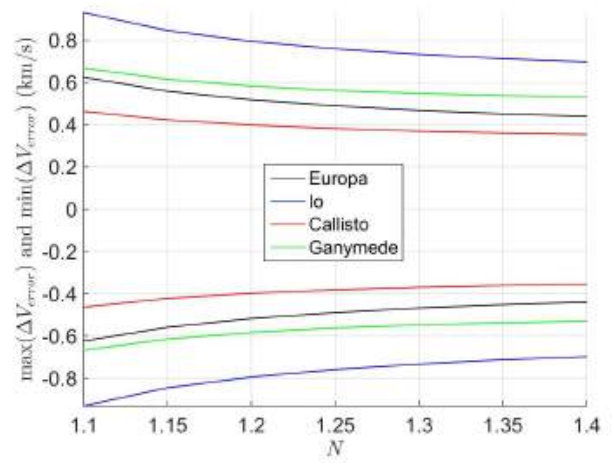

(c) $\Delta V_{\text {error }}$

Figure 3. Maximum and minimum $\Delta V \mathrm{~s}$ as function of $N$ applied to the Galilean moons. 


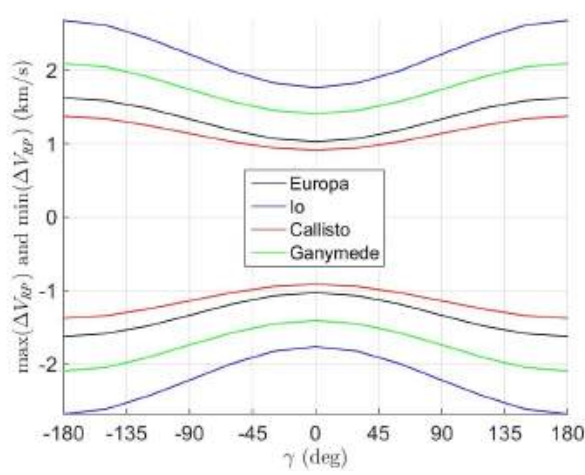

(a) $\Delta V_{R P}$

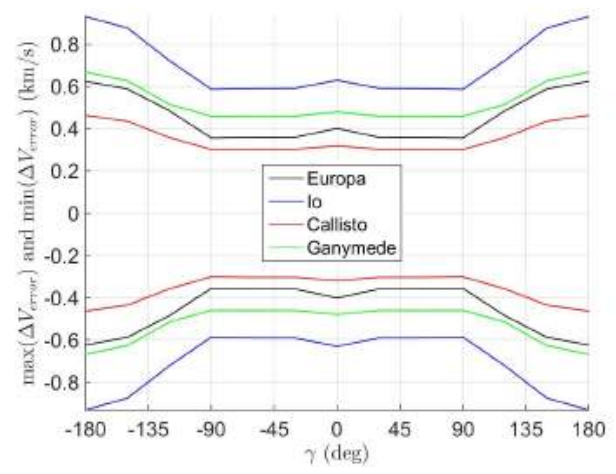

(c) $\Delta V_{\text {error }}$

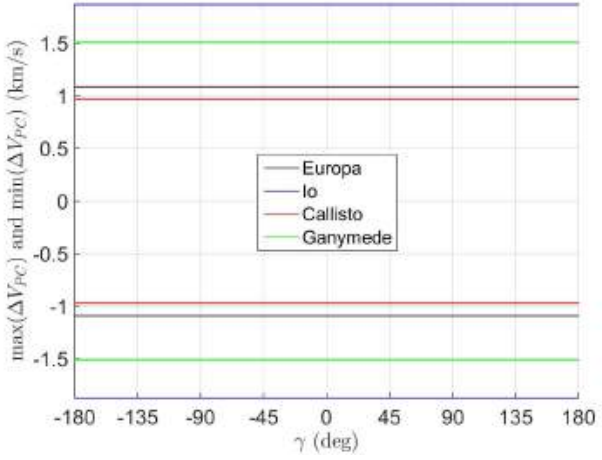

(b) $\triangle V_{P C}$

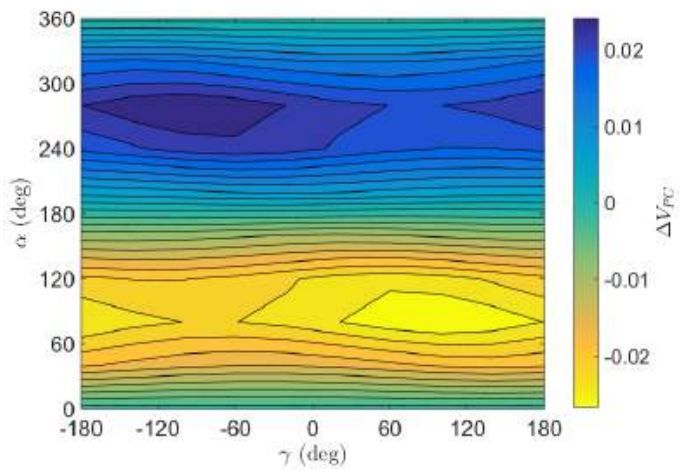

(d) $\Delta V_{P C}$ for $\beta=80^{\circ}, N=1.2, r_{p}=0.004$ and $\mu=$ $7.8 \times 10^{-5}$ (Ganymede-Jupiter) as function of $\alpha$ and $\gamma$.

Figure 4. Maximum and minimum $\Delta V \mathrm{~s}$ as function of $\gamma$ applied to the Galilean moons.

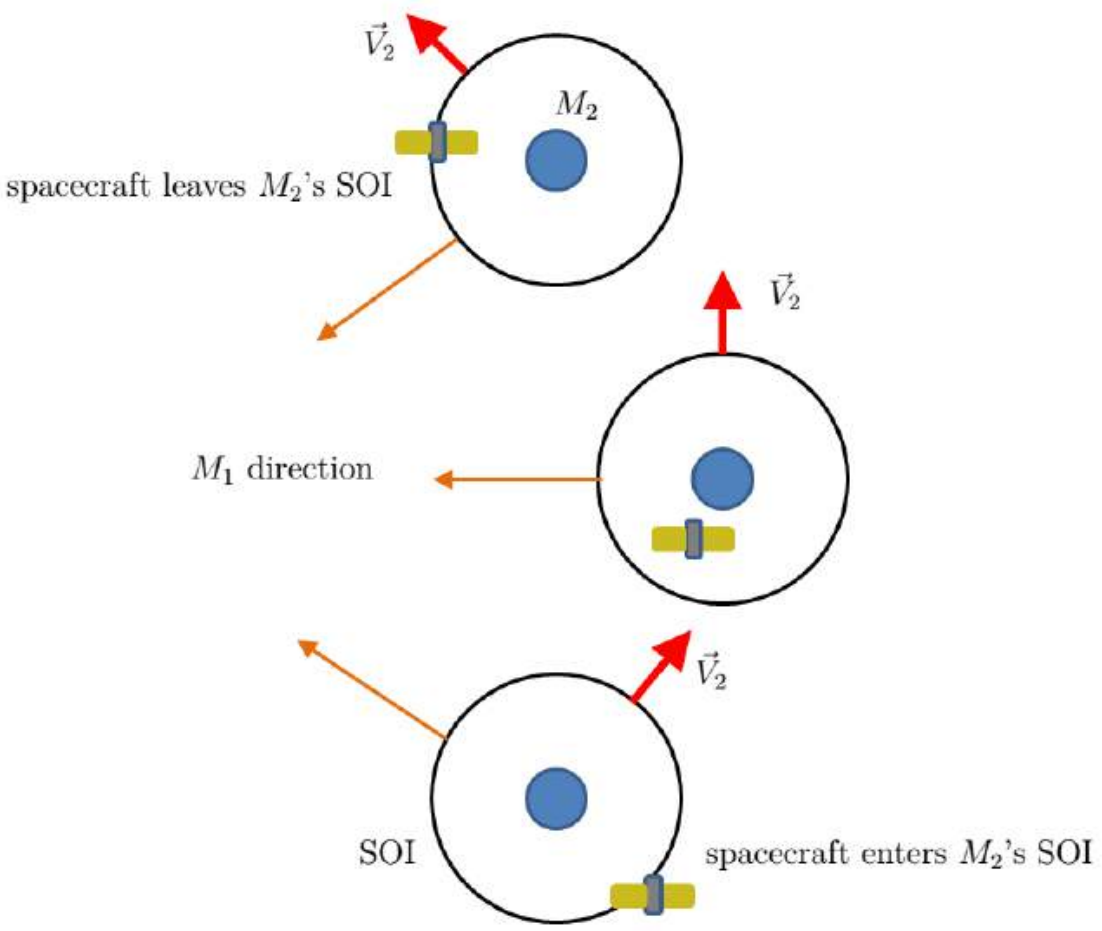

Figure 5. $M_{2}$ swing-by as seems from the inertial reference system $X Y Z$. 


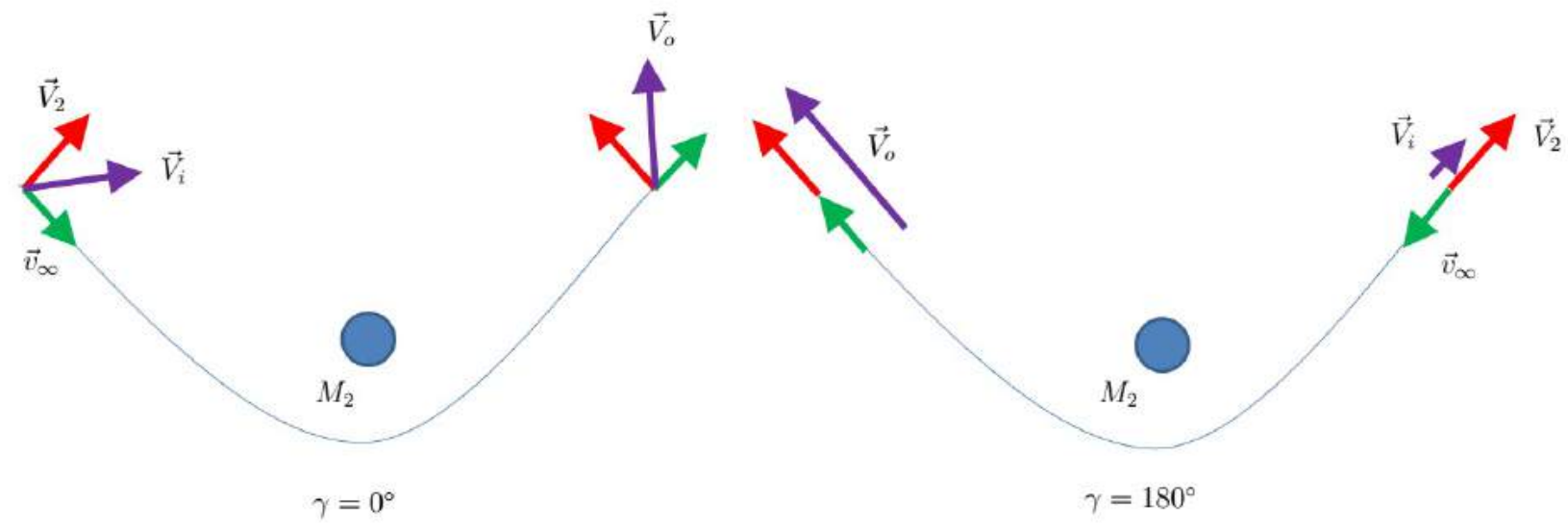

Figure 6. $M_{2}$ swing-by as seem from the pseudo-inertial reference frame $X^{\prime} Y^{\prime} Z^{\prime}$, and recognizing it as a non-inertial reference frame.

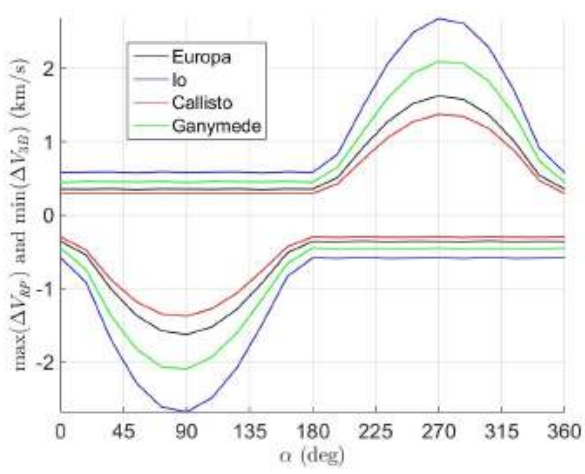

(a) $\Delta V_{R P}$

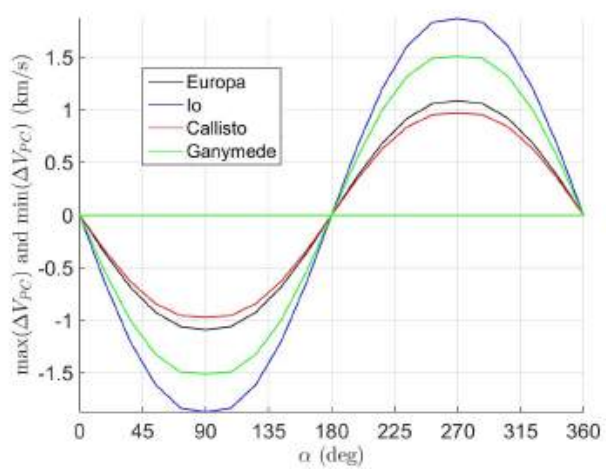

(b) $\Delta V_{P C}$

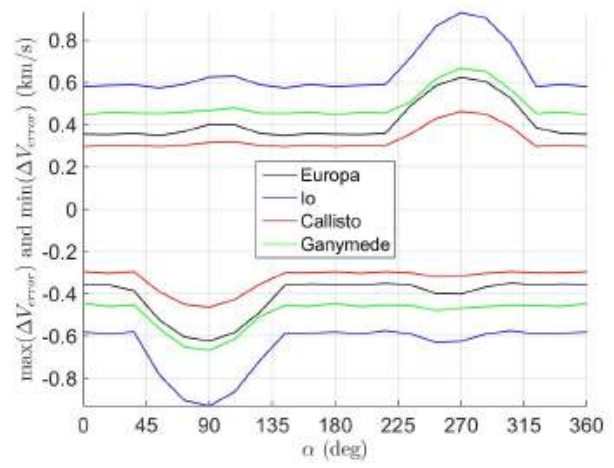

(c) $\Delta V_{\text {error }}$

Figure 7. Maximum and minimum $\Delta V \mathrm{~s}$ as function of $\alpha$ applied to the Galilean moons. 


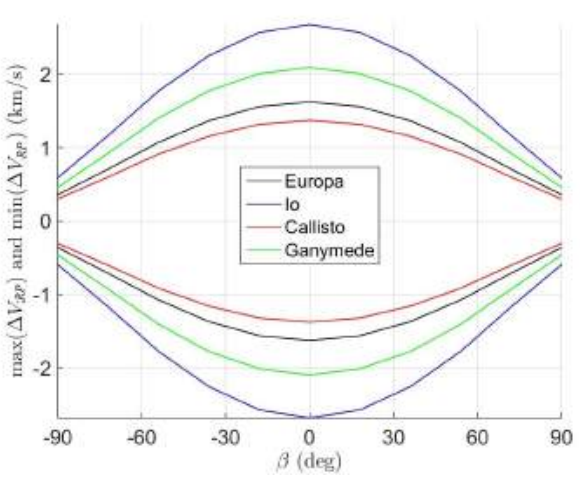

(a) $\Delta V_{R P}$

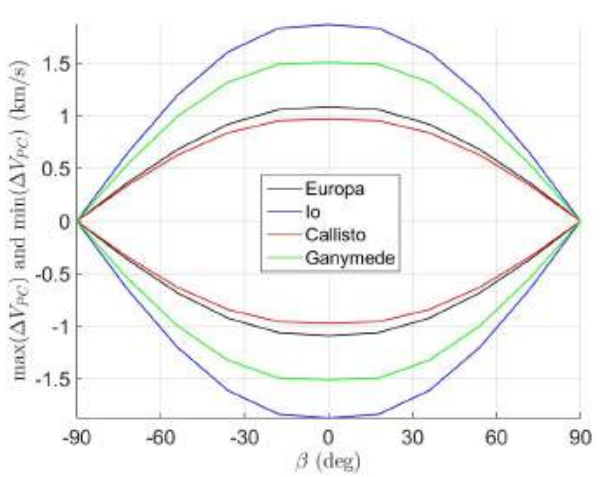

(b) $\triangle V_{P C}$

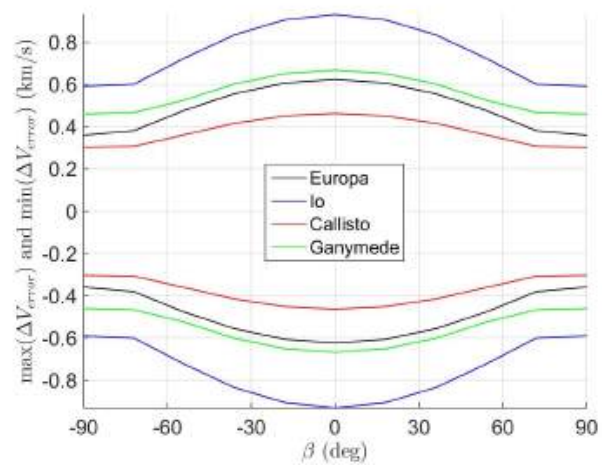

(c) $\Delta V_{\text {error }}$

Figure 8. Maximum and minimum $\Delta V$ s as function of $\beta$ applied to the Galilean moons.

\section{CONCLUSIONS}

This paper highlights the importance of taking a more realistic approach to design swing-bys applied to the Galilean moons, by showing that errors in $\Delta V$ s might arise up to $60 \%$ of the value estimated by the patched-conics approach.

Also, through the analysis of the $\gamma$ angle, the importance of the non-instantaneity of the maneuver, which is a consequence of considering the pseudo-inertial reference system as inertial, is highlighted.

The other results are in accordance with previous studies, as the ones made by Negri et al. (2017), Prado (2007) and Broucke (1988).

\section{ACKNOWLEDGEMENTS}

The authors thank the Grants \# 406841/2016-0 and 301338/2016-7 from the National Council for Scientific and Technological Development (CNPq), and Grants \# 2011/08171-3, 2011/13101-4, 2015/24321-6, 2016/14665-2 and 2016/186137 from São Paulo Research Foundation (FAPESP).

\section{REFERENCES}

Araujo, R., Winter, O., Prado, A. and Vieira Martins, R., 2008. "Sphere of influence and gravitational capture radius: a dynamical approach". Monthly Notices of the Royal Astronomical Society, Vol. 391, No. 2, pp. 675-684.

Bolton, S., 2010. "The juno mission". Proceedings of the International Astronomical Union, Vol. 6, No. S269, pp. 92-100.

Broucke, R., 1988. "The celestial mechanics of gravity assist". In Astrodynamics Conference. p. 4220.

Grasset, O., Dougherty, M., Coustenis, A., Bunce, E., Erd, C., Titov, D., Blanc, M., Coates, A., Drossart, P., Fletcher, L. et al., 2013. "Jupiter icy moons explorer (juice): An esa mission to orbit ganymede and to characterise the jupiter system". Planetary and Space Science, Vol. 78, pp. 1-21.

Greenberg, R., Carusi, A. and Valsecchi, G., 1988. "Outcomes of planetary close encounters: a systematic comparison of methodologies". Icarus, Vol. 75, No. 1, pp. 1-29.

Minovitch, M.A., 2010. "The invention that opened the solar system to exploration". Planetary and Space Science, Vol. 58, No. 6, pp. 885-892.

Murray, C.D. and Dermott, S.F., 1999. Solar system dynamics. Cambridge university press. 
Negri, R.B., de Almeida Prado, A.F.B. and Sukhanov, A., 2017. "Studying the errors in the estimation of the variation of energy by the "patched-conics" model in the three-dimensional swing-by". Celestial Mechanics and Dynamical Astronomy, pp. 1-16.

Phillips, C.B. and Pappalardo, R.T., 2014. "Europa clipper mission concept: Exploring jupiter's ocean moon". Eos, Transactions American Geophysical Union, Vol. 95, No. 20, pp. 165-167.

Prado, A., 2000. "An analytical description of the close approach maneuver in three dimensions". In 51th International Astronautical Congress.

Prado, A.F.B.d.A., 2007. "A comparison of the patched-conics approach and the restricted problem for swing-bys". Advances in Space Research, Vol. 40, No. 1, pp. 113-117.

Prado, A.F.B.d.A. and de Felipe, G., 2007. "An analytical study of the powered swing-by to perform orbital maneuvers". Advances in Space Research, Vol. 40, No. 1, pp. 102-112.

Szebehely, V., 1967. "Theory of orbits academic press new york google scholar".

\section{RESPONSIBILITY NOTICE}

The authors are the only responsible for the printed material included in this paper. 